\title{
Left ventricular pseudoaneurysm complicating acute inferoposterior myocardial infarction
}

\author{
Vijay Yadav*, Ratna Mani Gajurel, Chandra Mani Poudel, Manju Sharma and Suman Adhikari \\ Department of Cardiology, Manmohan Cardiothoracic Vascular and Transplant Center, Nepal
}

\section{Summary}

Left ventricular pseudoaneurysm is a rare mechanical complication of acute myocardial infarction. We describe a 60 -year-old male who presented with typical ischemic type of chest pain with low blood pressure and heart rate. A 12-lead electrocardiogram showed atrioventricular (AV) dissocation with ST segment elevation in the inferior leads. The initial echocardiography depicted moderate pericardial effusion without tamponade. The possibilities of both, acute mechanical complications of myocardial infarction, and acute aortic dissection were sought of. The echocardiography on the following day revealed a free wall rupture of the infero-posterior wall of left ventricle with formation of a pseudoaneurysm. This was further proved by the computed tomography (CT) of chest with contrast, which in addition, ruled out acute aortic dissection. The patient was planned for an early surgery. Pre-operative coronary angiogram revealed complete occlusion of distal left circumflex artery and 70-80\% discrete stenosis of mid left anterior descending artery.

\section{Background}

Although left ventricular pseudoaneurysm (LV-PsA) is a rare occurrence, its presence, however, signifies a poor prognosis and is known to be a lethal condition due to its higher propensity to get ruptured in comparison with the true aneurysm. The clinical presentation is non-specific, making the diagnosis challenging. Hence, a high index of suspicion is needed. Any diagnostic delay would exempt the patient from early surgical repair which is the mainstay of treatment.

\section{Case presentation}

A 60-year-old man, current cigarette smoker, presented in April 2020 to the emergency room with a sudden onset of severe retrosternal pain for four days duration that occurred even at rest. The pain radiated to his left arm and was associated with diaphoresis and one episode of vomiting. He was free of pain at the time of his arrival to our emergency room.

On physical examination, he looked anxious, was bradycardic ( 60 beats/min, regular) and hypotensive $(90 / 50 \mathrm{~mm} \mathrm{Hg}$ ). The jugular venous pressure was not elevated. The chest auscultation revealed bilateral crackles and the breath sounds were bilaterally equal. The heart sounds were normal without cardiac murmer or added sounds.

\section{Investigations}

His baseline renal function was impaired with serum urea and creatinine values of $76 \mathrm{mg} / \mathrm{dL}$ and $1.7 \mathrm{mg} / \mathrm{dL}$ respectively. His quantitative cardiac troponin I was raised, i.e.8.3ng/ml (normal:
$<0.012 \mathrm{ng} / \mathrm{ml}$ ). The baseline chest $\mathrm{x}$-ray showed cardiomegaly (Figure 1). A 12-lead left sided electrocardiogram (ECG) showed ST segment elevation $(>1 \mathrm{~mm})$ in lead III $>$ aVF $>$ II, ST segment depression in lead $\mathrm{aVL}>\mathrm{I}$, and ST segment depression $(>1 \mathrm{~mm}$ ) in leads V1-V4 with AV dissociation (Figure 2). A 12-lead right sided ECG revealed low voltage in RV5-RV6 with ST segment coving and T wave inversion (Figure 3) and the posterior ECG depicted ST segment elevation $(>1 \mathrm{~mm})$ in leads V7-V9 (Figure 4).

Bedside transthoracic echocardiography revealed moderate pericardial effusion without features of tamponade, absent intimal flaps, hypokinetic right coronary artery (RCA) and left circumflex artery (LCx) territories with an ejection fraction of $30 \%$, and moderate mitral regurgitation.

The possibilities of both mechanical complications of acute MI or acute aortic dissection were suspected and patient was planned for an emergency CT chest with contrast. It was however delayed due to baseline renal impairment (S. Creatinine: $1.7 \mathrm{mg} / \mathrm{dL}$ ).

The echocardiography (Figure 6) on the following day revealed a dyskinetic and thinned out LV inferoposterior wall with a free wall rupture and moderate pericardial effusion without tamponade

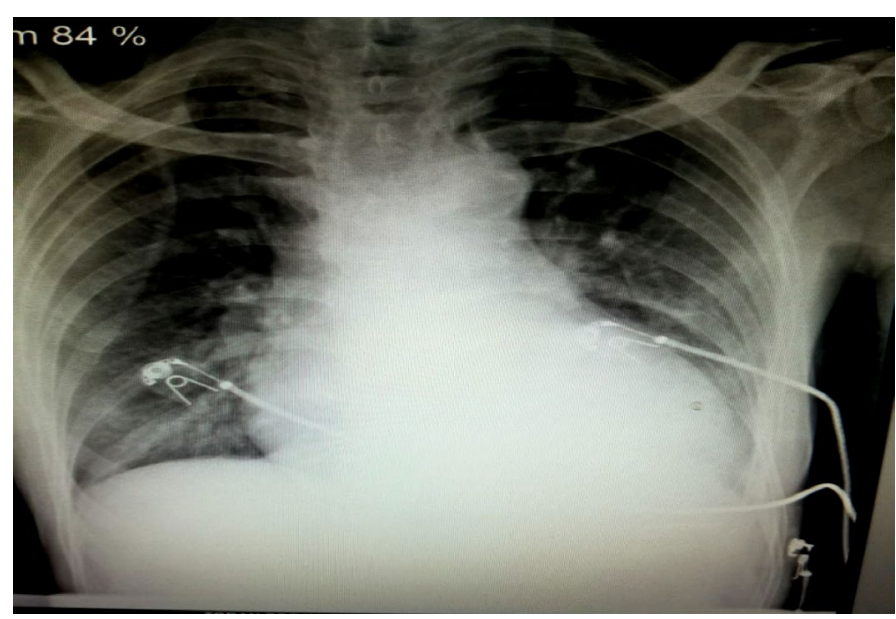

Figure 1. Chest $\mathrm{X}$ ray showing cardiomegaly

*Correspondence to: Vijay Yadav, Department of Cardiology, Manmohan Cardiothoracic Vascular and Transplant Center, Nepal, Tel: +977-9851201126, E-mail: vjmedicine451@gmail.com

Received: September 16, 2020; Accepted: September 28, 2020; Published: October 07,2020 


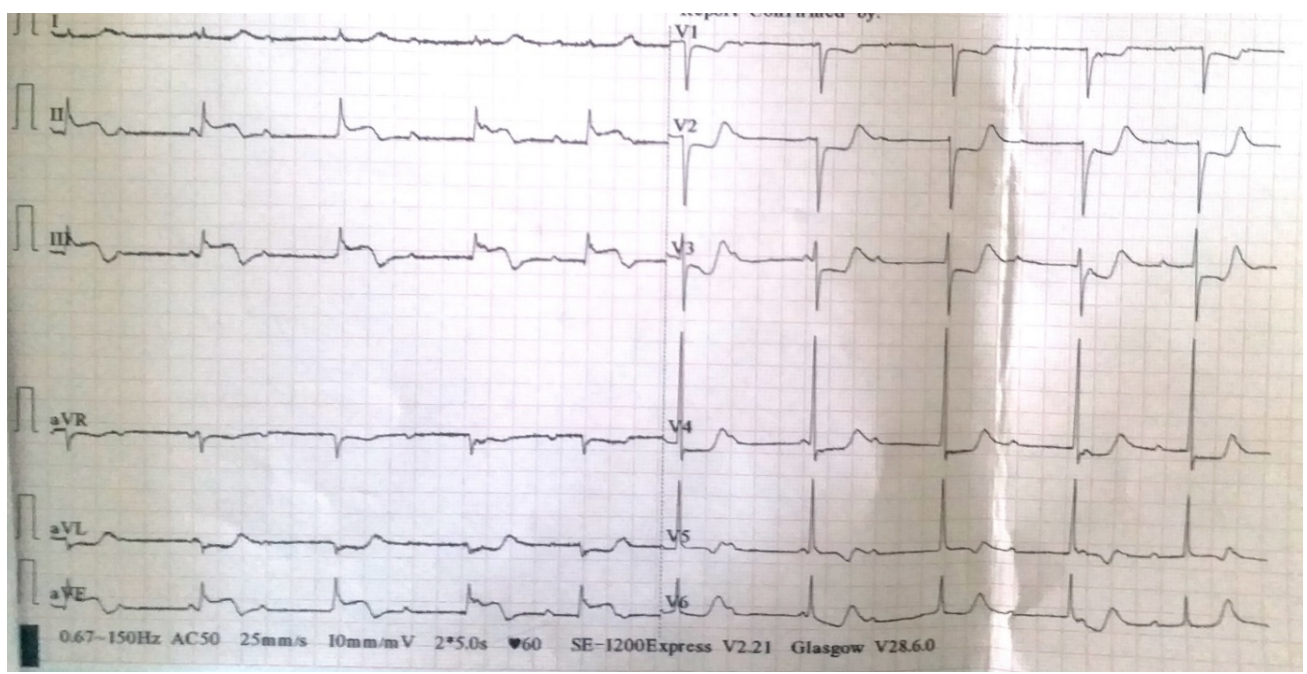

Figure 2. A 12-lead ECG showing ST elevation $(>1 \mathrm{~mm})$ in lead III $>$ aVF $>$ II, ST depression in lead aVL $>$ I, and ST depression $(>1 \mathrm{~mm})$ in leads V1-V4 with AV dissociation

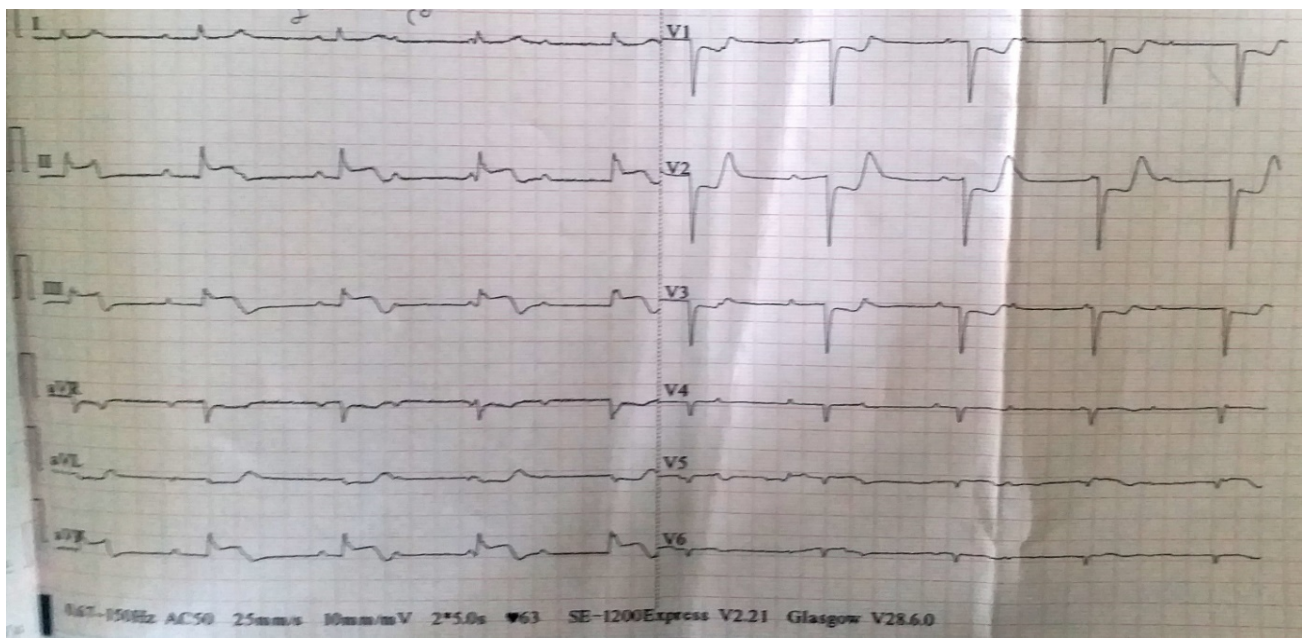

Figure 3. 12 lead right sided ECG showing low voltage in RV5-RV6 with ST coving and T wave inversion and AV dissociation

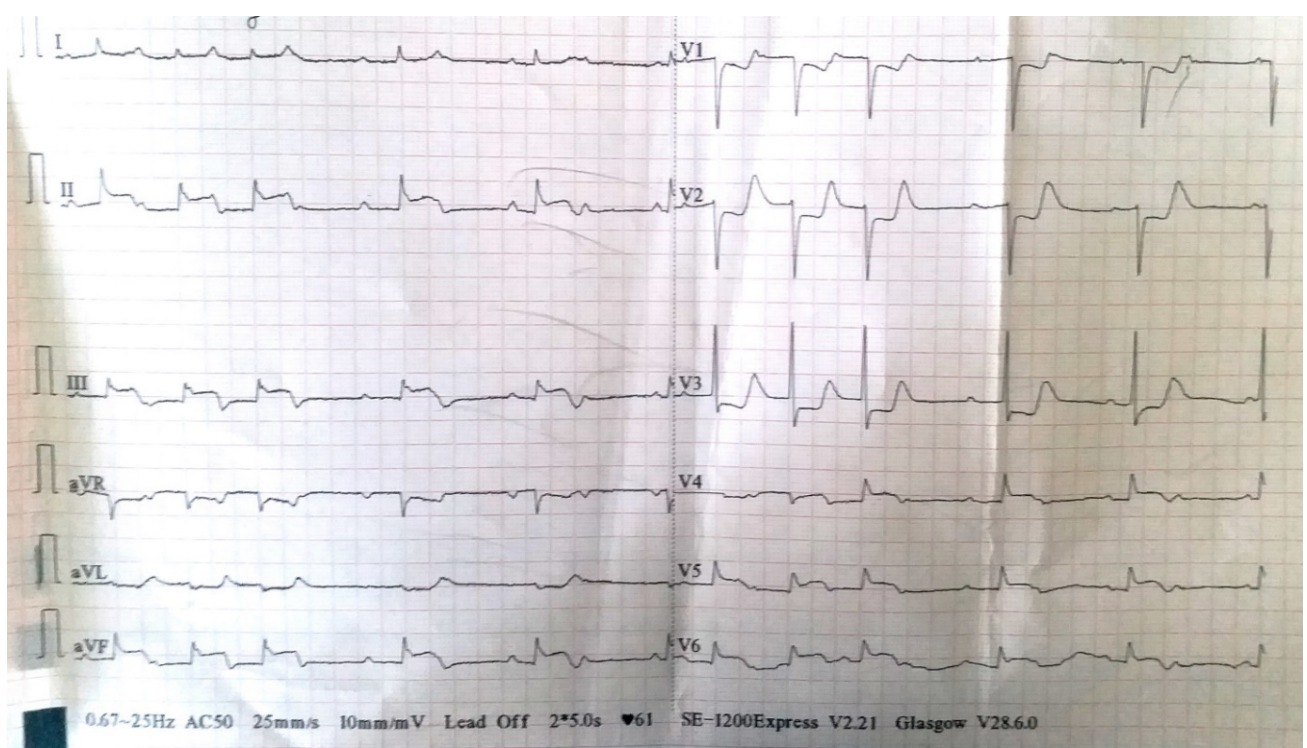

Figure 4. A posterior ECG showing ST elevation (> 1mm) in leads V7-V9 (represented on the ECG in leads V4-V6) and AV dissociation with intermittent junctional beats 


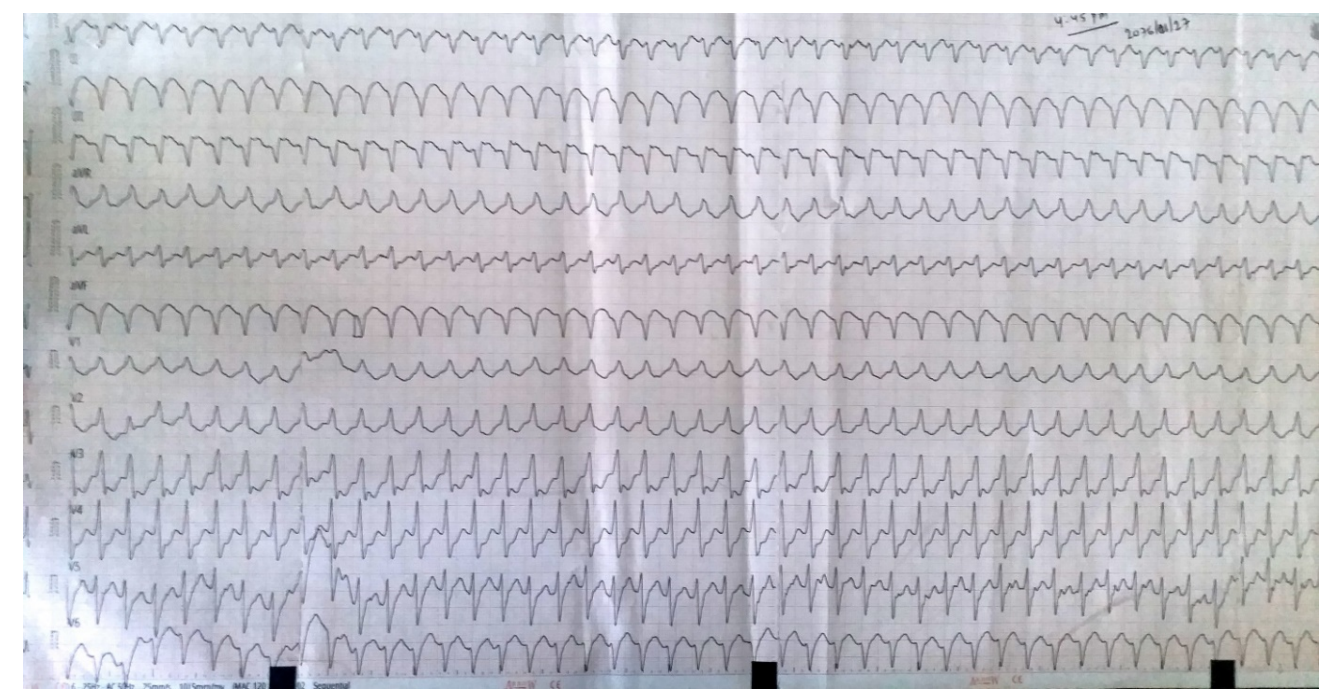

Figure 5. A 12 lead ECG showing RBBB morphology monomorphic VT with superior axis originating most probably from the infero-posterior wall of left ventricle
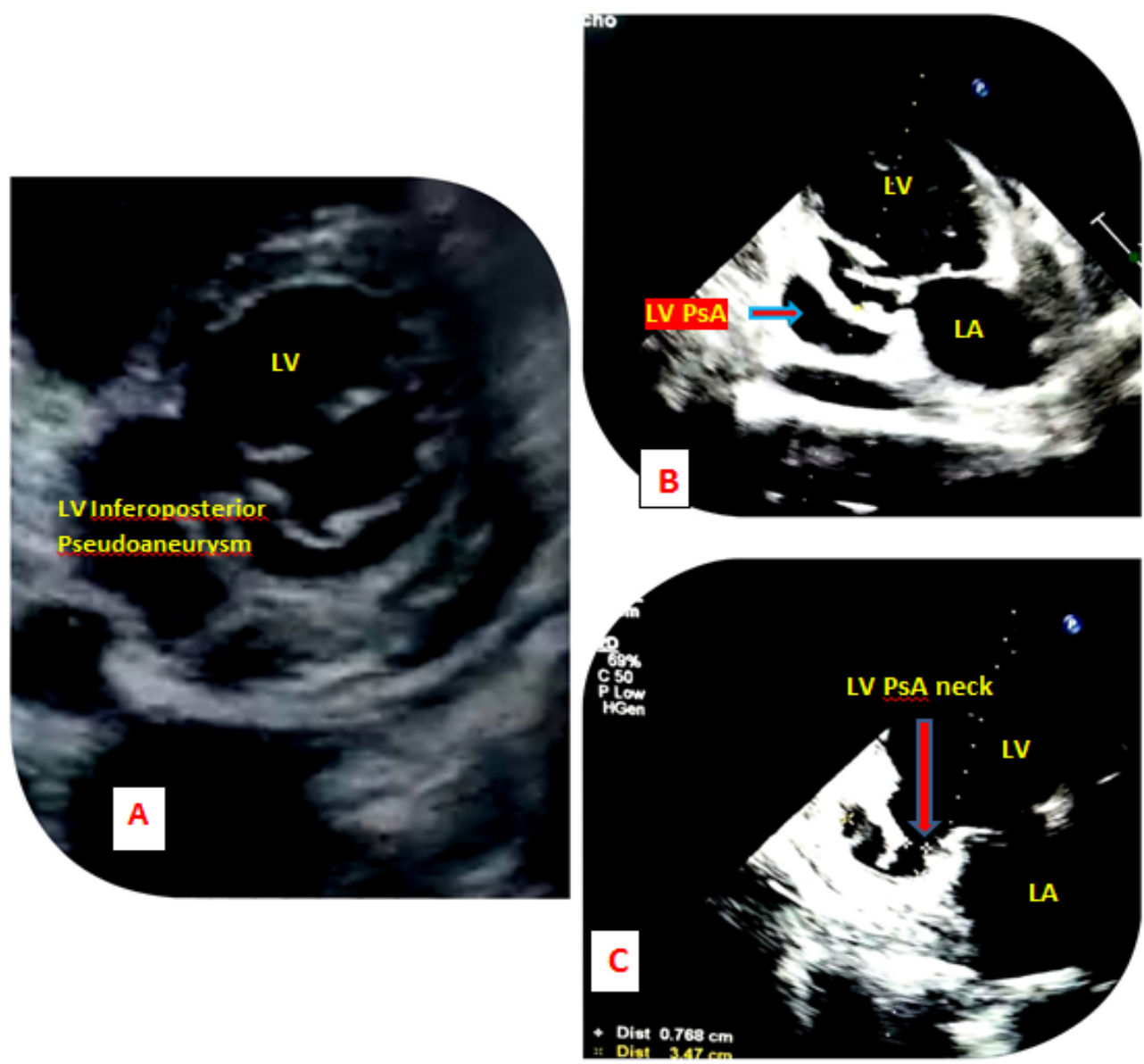

Figure 6. 2D transthoracic echocardiogram (A) Parasternal short axis view showing a spherical shaped LV cavity and a PsA involving the inferoposterior wall of LV. (B) Apical 2- chamber view showing LV PsA in the inferoposterior wall with a closed aneurysmal neck. (C) Apical 2-chamber view showing PsA involving the basal segments of inferoposterior wall with an open narrow neck $(7.6 \mathrm{~mm})$ and aneurysmal diameter $(34.7 \mathrm{~mm})$ with a neck diameter ratio of less than 0.5

physiology. A large abnormal cavity was visualized communicating with the posterior wall of the left ventricle. The width of the neck was $7.6 \mathrm{~mm}$ and the maximum internal diameter of the aneurysmal sac was $34.7 \mathrm{~mm}$ with a neck to sac ratio of less than 0.5 (about 0.22 ). Bidirectional shunt (Figure 7) was evident across the neck of the sac on the colour doppler which was strongly suggestive of a pseudoaneurysm. The ejection fraction was $30 \%$.

Upon stabilization of renal parameters, he underwent CT chest with contrast that depicted a LV posterior wall pseudoaneurysm with a neck and internal diameter of the aneurysmal sac sized $7.6 \mathrm{~mm}$ and 57 
mm respectively (Figure 9) with moderate pericardial and left pleural effusion. Additionally, it also ruled out acute aortic dissection.

Pre-operative coronary angiogram (CAG) was done which revealed double vessel disease (DVD) - complete occlusion of distal left circumflex artery and 70-80\% discrete stenotic lesion of the midleft anterior descending artery (Figure 8).
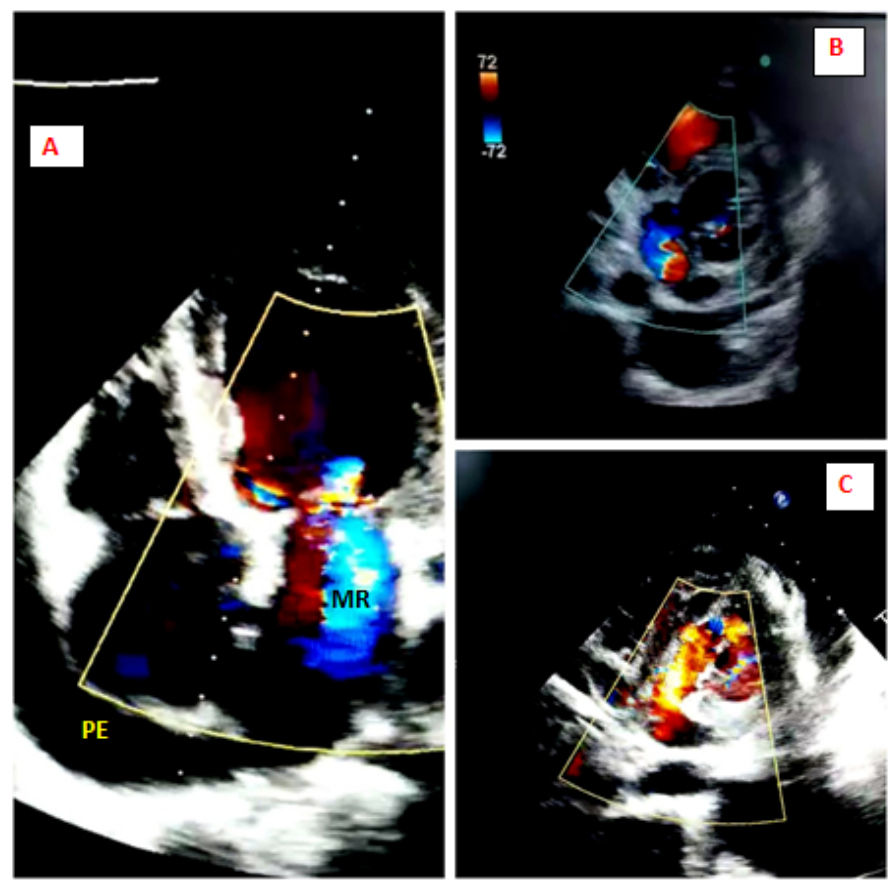

Figure 7. 2D transthoracic echocardiogram (A) Apical 4-chamber view showing moderate mitral regurgitation (MR) and moderate pericardial effusion (PE). (B and C) Parasternal short axis view showing a bidirectional shunt across the LV PsA showing a bidirectional shunt across the LV PsA

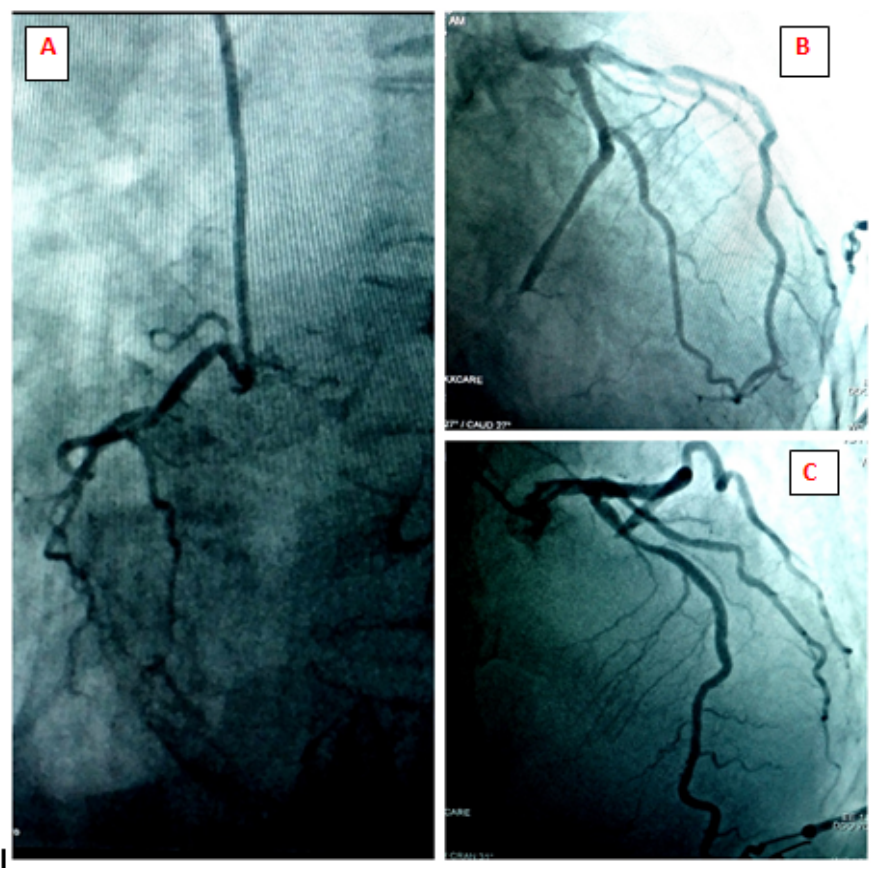

Figure 8. Coronary angiography: (A) Right coronary angiogram, LAO caudal projection showing a non-dominant right coronary artery. (B) Left coronary angiogram, RAO caudal projection showing complete occlusion of distal left circumflex artery. (C) Left coronary angiogram, RAO cranial view showing $70-80 \%$ discrete stenotic lesion of mid left anterior descending artery

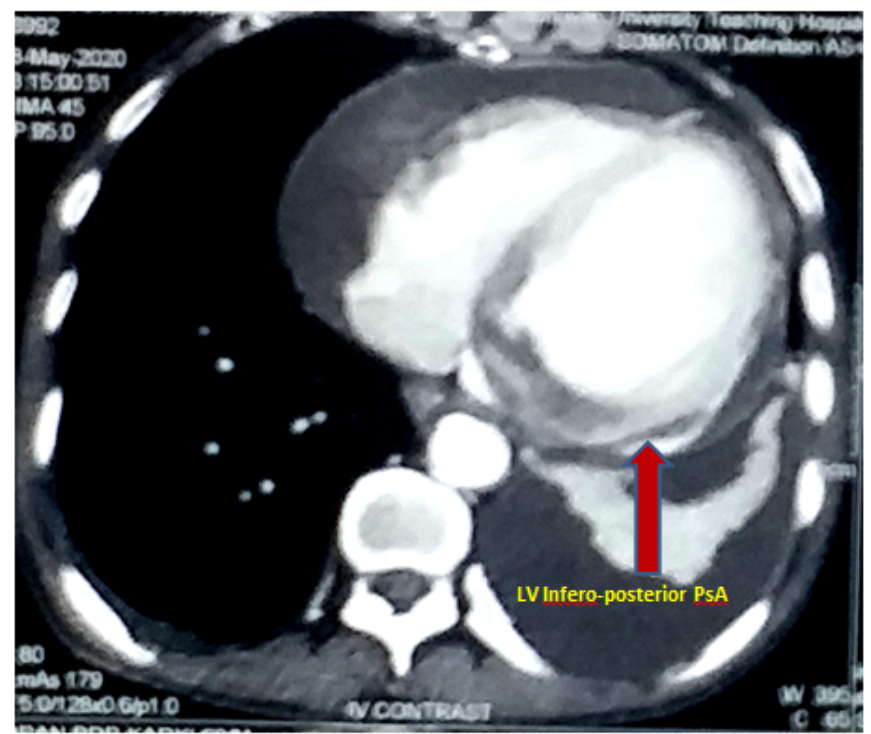

Figure 9. CECT chest showing LV inferio-posterior pseudoaneurysm

\section{Differential diagnosis}

Most of the pericardial effusion is presumed to be due to undiagnosed viral etiologies termed idiopathic. It can also be due to uremia, malignancy, autoimmune inflammatory diseases or long-term radiation therapy to mediastinum in the chronic settings. Acutely, pericardial effusion is a consequence of mechanical complications of MI (LV free wall rupture or pseudoaneurysm rupture), acute aortic dissection, chest wall trauma, and iatrogenic. The differential diagnosis was narrowed to either post-MI mechanical complications or acute aortic dissection.

\section{Treatment}

The patient was managed with dual antiplatelets, statin, ionotropes and other supportive measures. He developed an episode of RBBB morphology monomorphic ventricular tachycardia with superior axis originating most probably from the infero-posterior wall of left ventricle (Figure 5). It was reverted to sinus rhythm by the synchronized DC shock of 200 Joules and was kept on maintenance amiodarone. He was planned for an early surgery.

\section{Outcome and follow-up}

The patient developed right sided hospital acquired pneumonia while waiting for the surgery. The test for Corona Virus (SARS-CoV-2 RT-PCR) was negative. His clinical status took a downhill course and subsequently he was intubated and mechanically ventilated. The patient died on the ninth day of hospital admission.

\section{Discussion}

Left ventricular (LV) pseudoaneurysms (PSA) form when cardiac rupture is contained by adherent pericardium or scar tissue [1]. Unlike a true aneurysm, a PSA contains no endocardium or myocardium. Myocardial rupture usually occurs within the first 3-5 days after myocardial infarction (MI) in half of the cases (early rupture) and in the first two weeks in $90 \%$ (late rupture) [2]. The improved medical and the interventional management of acute $\mathrm{MI}$ have decreased the incidence of LV PSA. The incidence of LV PSA after MI is 0.2 to $0.3 \%$ [3]. The most common cause of LV PSA is transmural MI [4]. The inferior infarcts 
have approximately a two times higher incidence than anterior infarcts [1]. The mortality rate is as high as $40 \%$ with complications such as rupture, cardiac tamponade, thromboembolism, and arrhythmia [5]. The risk of rupture is as high as $30-45 \%$ if left untreated [6]. The inferior or inferolateral walls are the most common site of LV PSA and the risk factors for its development are advanced age, female sex, hypertension, first MI, lack of collaterals, late presentation of MI, and delayed or no revascularization [3].

The most frequently reported symptoms are congestive heart failure, chest pain, and dyspnea, but $>10 \%$ of patients are asymptomatic. A cardiac mumur is present in $70 \%$ of patients which is often to-andfro. In the electrocardiography, almost all patients have non-specific ST changes and only $20 \%$ have ST segment elevation. Evidence of mass on chest $\mathrm{X}$-ray is seen in more than $50 \%$ of patients which provides an important clue to the diagnosis [1]. The transthoracic echocardiogram is a reasonable first step, but a definitive diagnosis is made in only $26 \%$ of patients [7]. PSA communicates with the LV cavity through a narrow neck, with a diameter $<50 \%$ of the maximum internal dimensions of the aneurysm with a bidirectional flow in the color doppler.

The most reliable method for the diagnosis of LV PSA is LV angiography [8] which yields a definitive diagnostic accuracy in more than $85 \%$ of patients [1]. Cardiovascular computerized tomography (CT) and magnetic resonance imaging (MRI) are an alternative to angiography or echocardiography that may be useful in order to distinguish a pseudoaneurysm from a true aneurysm [9]. CT and MRI can further characterize the PSA site, size, extension, and anatomic relationship to the surrounding structures. Cardiac MRI has a sensitivity of $100 \%$ and specificity of $83 \%$ [10].

In our case, the definitive diagnosis was made by CT chest with contrast which additionally ruled out aortic dissection. Surgery is the primary modality of treatment. Compared to the medical management (which has a mortality rate of $48 \%$ ), surgical therapy has a mortality rate of $23 \%$ [3].

\section{Learning points/take home messages}

1. The inferior infarcts have approximately two times higher incidence of pseudoaneurysm than anterior infarcts.

2. LV pseudoaneurysm is an extremely lethal condition with the mortality rate of $40 \%$ and the rate of spontaneous rupture is $30-45 \%$ if left untreated.
3. High clinical suspicion and early utilization of non-invasive modalities for its detection are essential to improve the outcome of the patient.

4. The systolic murmur is absent in $30 \%$ of cases.

5. Transthoracic echocardiography is an acceptable first line test to detect pseudoaneurysm. Cardiac CT and MRI are the other alternative non-invasive diagnostic tests.

6. Medical therapy only has a mortality rate of $48 \%$.

7. Surgical therapy is the mainstay of treatment which has a mortality rate of $23 \%$.

\section{References}

1. Frances C, Romero A, Grady D (1998) Left ventricular pseudoaneurysm. J Am Coll Cardiol 32: 557-561. [Crossref]

2. Marchandot B, Crimizade U, El Ghannudi S, Morel O (2018) Giant ventricular pseudoaneurysm following inferior myocardial infarction: insights from multimodal imaging approach. Eur Heart J Case Rep 2: yty019. [Crossref]

3. Al Saidi K, Malik SA, Albulushi A, Moulton M, Chatzizisis YS (2019) Left ventricular pseudoaneurysm complicated with very late rupture 5 years after myocardial infarction. JACC Case Rep 1: 569-572.

4. Prifti E, Bonacchi M, Baboci A, Giunti G, Veshti A, Demiraj A, et al. (2017) Surgical treatment of post-infarction left ventricular pseudoaneurysm: case series highlighting various surgical strategies. Ann Med Surg 16: 44-51. [Crossref]

5. Hulten EA, Blankstein R (2012) Pseudoaneurysms of the heart. Circulation 125: 19201925. [Crossref]

6. Webb J, Gemmell M, Al-Fakih K, Chiribiri A (2016) Medical treatment of left ventricular pseudoaneurysms. QJM: Int J Med 109: 213-214.

7. Kupari M, Verkkala K, Maamies T, Härtel G (1987) Value of combined cross sectional and Doppler echocardiography in the detection of left ventricular pseudoaneurysm after mitral valve replacement. Br Heart J 58: 52-56 [Crossref]

8. Al-Saadon K, Walley V, Green M, Beanlands D (1995) Angiographic diagnosis of true and false LV aneurysms after inferior wall myocardial infarction. Cathet Cardiovasc Diagn 35: 266-269. [Crossref]

9. Konen E, Merchant N, Gutierrez C, Provost Y, Mickleborough L, Paul NS, et al. (2005) True versus false left ventricular aneurysm: differentiation with MR imaging-initial experience. Radiology 236: 65-75. [Crossref]

10. Gill S, Rakhit D, Ohri S, Harden S (2011) Left ventricular true and false aneurysms identified by cardiovascular magnetic resonance. $\mathrm{Br} J$ Radiol 84: e035-e 037 . [Crossref]

Copyright: (C2021 Yadav V. This is an open-access article distributed under the terms of the Creative Commons Attribution License, which permits unrestricted use, distribution, and reproduction in any medium, provided the original author and source are credited. 